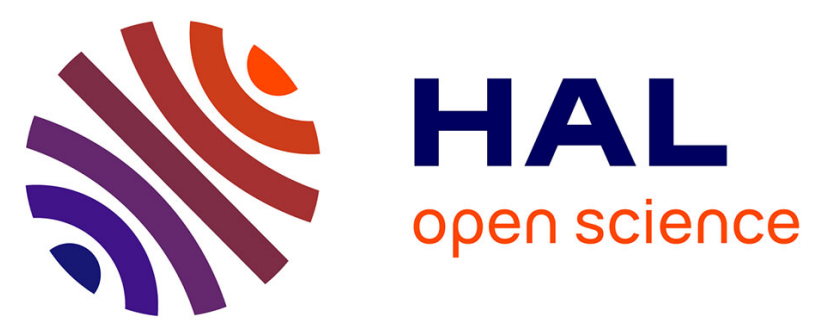

\title{
La coupe de l'oued Ahmadi (Dahar, sud tunisien): son intérêt pour la compréhension de l'évolution climatique au Pléistocène moyen
}

\author{
Jean-Louis Ballais, H. Ben Ouezdou, M. Ghanmi, K. Regaya
}

\section{To cite this version:}

Jean-Louis Ballais, H. Ben Ouezdou, M. Ghanmi, K. Regaya. La coupe de l'oued Ahmadi (Dahar, sud tunisien): son intérêt pour la compréhension de l'évolution climatique au Pléistocène moyen. Méditerranée: revue géographique des pays méditerranéens, 1988, 64 (2), pp.52-60. 10.3406/medit.1988.2549 . hal-01562378

\section{HAL Id: hal-01562378 \\ https://hal-amu.archives-ouvertes.fr/hal-01562378}

Submitted on 14 Jul 2017

HAL is a multi-disciplinary open access archive for the deposit and dissemination of scientific research documents, whether they are published or not. The documents may come from teaching and research institutions in France or abroad, or from public or private research centers.
L'archive ouverte pluridisciplinaire HAL, est destinée au dépôt et à la diffusion de documents scientifiques de niveau recherche, publiés ou non, émanant des établissements d'enseignement et de recherche français ou étrangers, des laboratoires publics ou privés. 
Jean-Louis Ballais

H. Ben Ouezdou

M. Ghanmi

K. Regaya

\section{La coupe de l'oued Ahmadi (Dahar, sud tunisien) : son intérêt pour la compréhension de l'évolution climatique au Pléistocène}

\section{moyen}

In: Méditerranée, Troisième série, Tome 64, 2-1988. Évolution des paysages tunisiens au cours du Quaternaire. pp. 52-60.

\section{Résumé}

La coupe de la terrasse du Pléistocène moyen de l'oued Ahmadi est organisée en quatre séquences sédimentaires qui pourraient traduire l'existence de quatre oscillations climatiques dans un climat restant aride dans l'ensemble. La genèse d'une croûte calcaire postérieurement à celle d'une croûte gypseuse indique un renversement épisodique de la tendance générale à l'aridification du climat pendant le Quaternaire.

\section{Abstract}

Four sedimentary sequences are seen in the Middle Pleistocene terrace of Wadi Ahmadi. They may indicate four climatic oscillations while climate remains arid for the most part. An occasional changing of direction in the general Quaternary trend to aridification is indicated by the genesis of a calcrust after a gypscrust.

Citer ce document / Cite this document :

Ballais Jean-Louis, Ben Ouezdou H., Ghanmi M., Regaya K. La coupe de l'oued Ahmadi (Dahar, sud tunisien) : son intérêt pour la compréhension de l'évolution climatique au Pléistocène moyen. In: Méditerranée, Troisième série, Tome 64, 2-1988. Évolution des paysages tunisiens au cours du Quaternaire. pp. 52-60.

doi : $10.3406 /$ medit.1988.2549

http://www.persee.fr/web/revues/home/prescript/article/medit_0025-8296_1988_num_64_2_2549 
La coupe de l'oued Ahmadi

(Dahar, sud tunisien) :

son intérêt pour la compréhension

de l'évolution climatique au Pléistocène moyen

\author{
J.-L. BALLAIS * \\ H. BEN OUEZDOU * * \\ M. GHANMI ** * \\ K. REGAYA * *
}

\begin{abstract}
Résumé - La coupe de la terrasse du Pléistocène moyen de l'oued Ahmadi est organisée en quatre séquences sédimentaires qui pourraient traduire l'existence de quatre oscillations climatiques dans un climat restant aride dans l'ensemble. La genèse d'une croûte calcaire postérieurement à celle d'une croûte gypseuse indique un renversement épisodique de la tendance générale à l'aridification du climat pendant le Quaternaire.
\end{abstract}

Abstract - Four sedimentary sequences are seen in the Middle Pleistocene terrace of Wadi Ahmadi. They may indicate four climatic oscillations while climate remains arid for the most part. An occasional changing of direction in the general Quaternary trend to aridification is indicated by the genesis of a calcrust after a gypscrust.

L'évolution climatique au Quatemaire dans le Sud tunisien a été généralement présentée comme une tendance à l'aridification (COQUE, 1962 ; BEN OUEZDOU, 1986, 1987). Cette position tient, en premier lieu, à la constatation que, selon les régions, aux périodes de formation de croûte calcaire (Pléistocène inférieur et moyen) succèdent des périodes de développement de croûte gypseuse (Pléistocène moyen ou supérieur et Holocène). L'absence de tout type de croûte à partir de l'Holocène moyen renforcé l'idée de l'accroissement de l'aridite jusqu'à la période actuelle. A cet égard, la coupe de l'oued Ahmadi dans une terrasse est significative car elle présente des éléments qui ne s'accordent pas avec cette hypothèse. En effet, il a été signalé récemment (BAL LAIS, 1984) la superposition d'une croûte calcaire à une croûte gypseuse. Ceci rompt le schéma classique connu dans la succession des types de croûte du Maghreb oriental et permet de nouvelles interprétations concemant l'évolution climatique au Pléistocène.

\title{
I - LES ENSEMBLES LITHOSTRATIGRAPHIQUES QUATERNAIRES DE L'OUED AHMADI
}

L'oued Ahmadi est un des oueds importants qui coulent sur le revers du monoclinal du Dahar, dans .e Sud tunisien (fig. 1). Il draine la partie septentrionale d'est en ouest, en direction des Grands Chotts tunisiens. Il s'encaisse dans le calcaire du Crétacé supérieur (Campanien) qui constitue le substratum des formations quaternaires. Ce calcaire campanien qui accuse un léger pendage général vers l'ouest, avec quelques replis d'extension très réduite (fig. 2).

A $20 \mathrm{~km}$ à l'ouest de Tamezret, l'oued Ahmadi et ses affluents montrent un ensemble de trois terrasses emboîtées (fig. 2 et 3). La haute terrasse est caractérisée par des dépôts grossiers passant à des limons gypseux et à nodules calcaires et se terminant par une croûte calcaire ou gypseuse. La moyenne terrasse s'emboîte dans la haute avec une très faible dénivellation. Il s'agit d'un dépôt limoneux avec quelques lentilles grossières. Des concrétions et une croûte calcaires, cette dernière peu épaisse et discontinue, caractérisent ces limons. Une basse terrasse peu étendue s'emboîte dans l'ensemble des deux terrasses antérieures : elle correspond à un dépôt hétérométrique à structure lenticulaire.

\footnotetext{
* Faculté des Sciences humaines et sociales, Bd 9 Avril, Tunis, Tunisie.

** Centre des Sciences de la terre, I.N.R.S.T., B.P. 95 Hammam Lif 2050, Tunisie.

*** Projet de cartographie góologique du Sud tunisien, O.N.M., B.P. 80, Gabès, Tunisie.
} 
Quelques éléments de datation permettent de placer chronologiquement ces terrasses. Leur âge quaternaire repose sur l'absence de tout dépôt tertiaire dans le plateau des Matmata et sa bordure occidentale (COQUE, 1962 ; ZOUARI et al., 1986 ; GHANMI, sous presse). Un pédiment à croûte calcaire et une terrasse à dépôt grossier ont été identifiés sur le revers du Dahar et attribués par COQUE (1962) respectivement au Villafranchien et au Quaternaire moyen. Sur la haute terrasse, nous avons recueilli des silex taillés du Paléolithique moyen (1) qui autorisent à placer la formation de cette terrasse au Pléistocène moyen. La moyenne et la basse terrasse dateraient du Pléistocène supérieur à la période historique.

Ainsi apparaît la complexité de cette terrasse du Pléistocène moyen qui en justifie une étude détaillée.

\section{II - DESCRIPTION DE LA COUPE DE LA TERRASSE DU PLEISTOCENE MOYEN}

\section{A. Les quatre séquences}

Les formations constituant la terrasse du Pléistocène moyen s'organisent en quatre séquences sédimentaires. La première séquence comprend, de bas en haut :

$-80 \mathrm{~cm}$ visibles de conglomérat hétérométrique constitué de cailloux et de quelques blocs subanguleux de calcaire campanien, dans une matrice limoneuse carbonatée et très peu sulfatée (OL 1, fig. 4), blanchâtre à rose (5YR 8/4), légèrement consolidée ; vers le haut, les éléments deviennent plus fins et apparaissent des poches de limons gypseux ;

$-25 \mathrm{~cm}$ de conglomérat (OL 2) à cail-

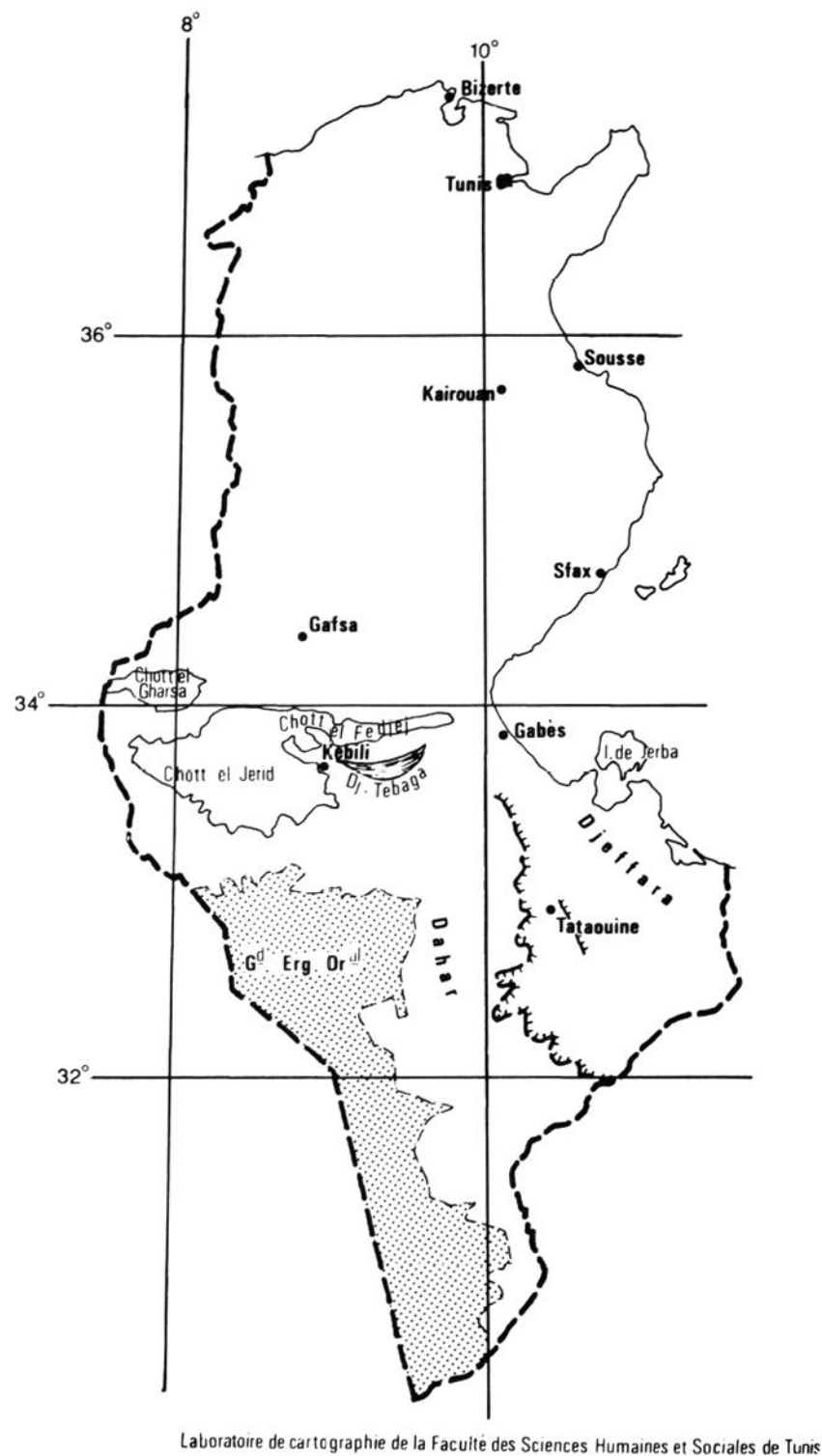

Fig. 1 : CARTE DE LOCALISATION loux plus petits, à matrice beige ( 5 YR 7/8) très gypseuse (le second maximum de $\mathrm{SO}_{3}$ de toute la coupe), plus pauvre en silice (fig. 4) ;

$-70 \mathrm{~cm}$ de limons sableux (OL 3), saumons (5YR 5/8), à concrétions gypseuses, structurés en prismes qui montent jusqu'à la croûte calcaire (OL 8). Ils ont une composition géochimique originale marquée, en particulier, par les maxima de $\mathrm{SiO}_{2}, \mathrm{Fe}_{2} \mathrm{O}_{3}$ et $\mathrm{Al}_{2} \mathrm{O}_{3}$ et le minimum de $\mathrm{CaO}$ (fig. 4). En lame mince, le squelette est essentiellement formé de grains de quartz qui représentent plus de $60 \%$ de la roche totale. Ces quartz forment deux stocks de taille et de forme différentes. Les premiers ont un diamètre moyen de l'ordre de $80 \mu \mathrm{m}$ et sont de forme arrondie et craquelée ; les seconds, dominants, ont un diamètre moyen de l'ordre de 40 à $50 \mu \mathrm{m}$ et ont une forme quelconque. Au fort grossissement (200 fois), ces quartz présentent des bordures partiellement dentelées, faisant apparaître des golfes de corrosion. Sur la bordure, plus ou moins intacts, existent des enduits ou des reliques d'argiles et/ou d'oxydes de fer. A côté, il existe des grains isolés de sparite de forme arrondie et de taille supérieure à celle des grains de quartz puisqu'ils dépassent facilement les $200 \mu \mathrm{m}$ de diamètre. Il s'agit vraisemblablement des restes de fragments de tests d'Inocérames, connus dans les séries crétacées. Des feldspaths sont également présents, mais rares (moins de $1 \%$ ), de forme plus ou moins arrondie. La glauconie représente 3 à $4 \%$ de l'ensemble du squelette ; elle s'individualise en grains arrondis noyés dans le plasma ; les grains verts sont rares, les oxydés plus nombreux et de petite taille ( $30 \mu \mathrm{m}$ de diamètre moyen). Le plasma est argilo-micritique, de 

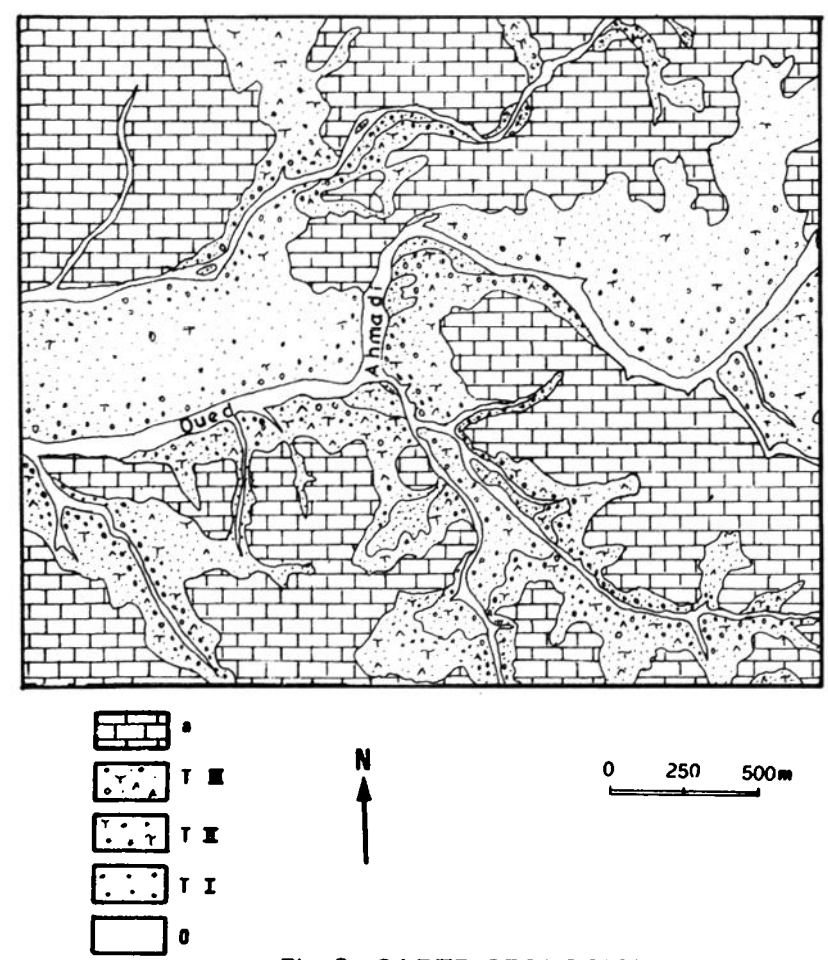

Fig. 2 : CARTE GEOLOGIQUE

a : calcaire campanien - TIII : haute terrasse - T II : moyenne terrasse - $\mathrm{Tl}$ : basse terrasse - 0 : lit majeur de l'oued Ahmadi. couleur brune à grise, dans lequel les grains de squelette sont épars, sans aucune structure visible. Ce matériel rappelle tout à fait les limons de Matmata (REGAYA, 1983).

$-45 \mathrm{~cm}$ de limons sableux encore gypseux (OL 4), saumons clairs (5YR 7/6), dans lesquels s'individualisent des nodules calcaires farineux clairs, dans une matrice à légère tendance grumuleuse. A côté du squelette et du plasma comparables à ceux décrits pour les limons précédents, on remarque une importante imprégnation gypseuse liée, à la fois, au squelette quartzique lui-même et à la présence des pores au sein du limon. En effet, le premier stock gypseux est strictement associé aux grains de quartz, il se présente en cristaux ou baguettes allongées et fusiformes, semblables aux "pattes de pie». Les cristaux, d'une longueur variable entre 80 et $100 \mu \mathrm{m}$ et d'une largeur ne dépassant guère les $10 \mu \mathrm{m}$, sont également noyés dans le plasma. Le second stock forme des plages plurimillimétriques "striées» par leurs clivages caractéristiques, ou alors de grosses baguettes allongées et fusiformes ( 2 à $3 \mathrm{~mm}$ de long et 0,5 à $1 \mathrm{~mm}$ de large). Ce gypse, qui occupe les pores, peut emprisonner des grains de squelette isolés ou même des plages tout entières de limons.

$-25 \mathrm{~cm}$ de sables limoneux de même structure (OL 5), gypseux, très siliceux, beiges (5YR 7/6) à nodules calcaires durs ;

$-60 \mathrm{~cm}$ de limons beige clair (OL 6) (5RY 7/6), à structure motteuse, contenant quelques concrétions calcaires, gypseux (fig. 4) et pauvres en $\mathrm{SiO}_{2}, \mathrm{Fe}_{2} \mathrm{O}_{3}$ et $\mathrm{Al}_{2} \mathrm{O}_{3}$. Cette augmentation du gypse a engendré : -une diminution du squelette de la roche originale (il ne représente plus que $50 \%$ ),

-un découpage du limon (squelette et plasma) lui-même en nodules séparés les uns des autres par du gypse recristallisé. On retrouve aussi les reliques de roches crétacées ou même de croûtes calcaires.

La deuxième séquence se réduit à deux dépôts :

- $50 \mathrm{~cm}$ de limons blanchâtres (OL 7) (5YR 8/3), à composition géochimique très proche de celle de OL 4 (fig. 4), contenant des lentilles de galets subanguleux de calcaire campanien, recouverts d'une pellicule calcaire sur l'ensemble de la surface et d'une concentration de gypse à la face inférieure. Le microfaciès de cet encroûtement comprend des structures diverses reflétant des zones de différents stades d'évolution (induration). Si l'on excepte le lit formé de la calcite sparitique qui permet la transition entre le galet et l'encroûtement même,

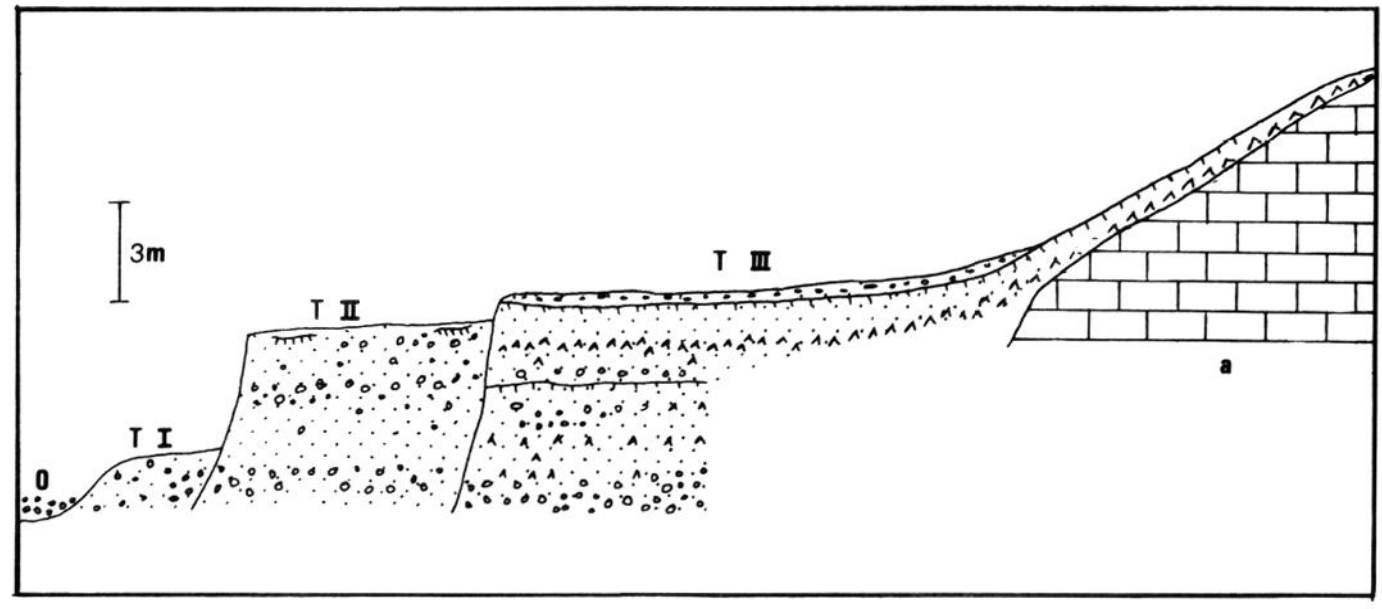

Laboratoire de carlographic de le Faculté des Sciences Humaines et Sociales de Tunis

Fig. 3 : COUPE SYNTHETIQUE DES TERRASSES DE L'OUED AHMADI 

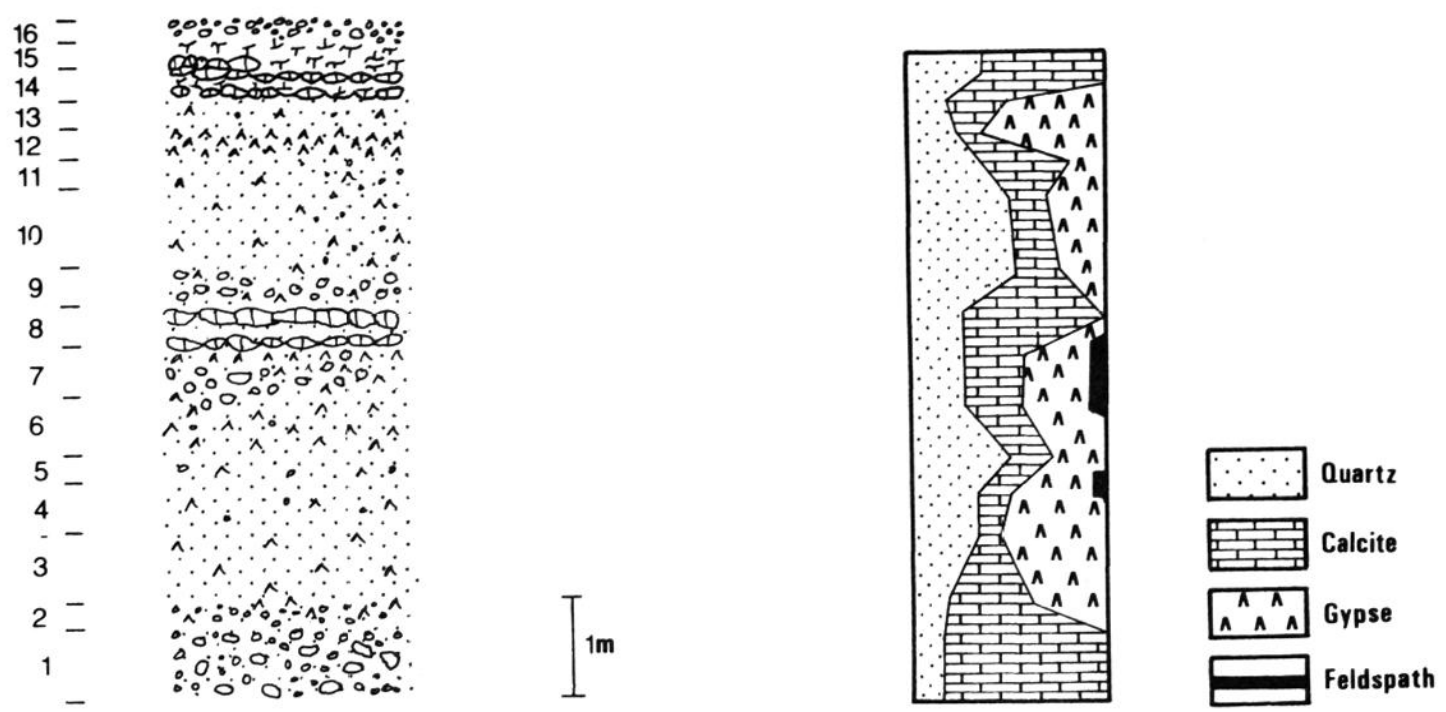

laboratoire de cartographie de la Faculté des Sciences Humaines et Sociales de Tunis

Fig. 4 : COUPE DE LA HAUTE TERRASSE DE L'OUED AHMADI

on retient trois principaux microfaciès décrits depuis les structures les plus indurées, du côté du galet, vers les structures friables les plus externes (fig. 5):

-une frange millimétrique de micrite brune sombre. Elle contient des grains de squelette non craquelés, de taille variant entre 30 et $50 \mu \mathrm{m}$; leurs bordures sont, le plus souvent, dentelées et remplacées par de la micrite ou de la microsparite ; quelques grains de quartz sont totalement remplacés par cette microsparite et ne subsiste que le fantôme du grain originel. Plusieurs grains de quartz gardent des enduits ou des reliques d'argiles et/ou d'oxydes de fer dans leurs golfes de corrosion. La glauconie est rare, mais elle subsiste ;

-un horizon plus clair que le précédent, avec un squelette identique et, toutefois, l'apparition d'un certain nombre de reliques de roches anciennes (tests de Lamellibranches, fragments de roches...), des nodules et, surtout, de la microsparite et de la sparite palissadique qui dessinent des formes plus ou moins complexes ;

l'horizon le plus friable est constitué de grains de squelette décrits plus haut, mais ils sont noyés dans un plasma micritique à microsparitique clair. La microsparite est orientée, elle est diffusée et peut englober des micronodules micritiques sans noyau.

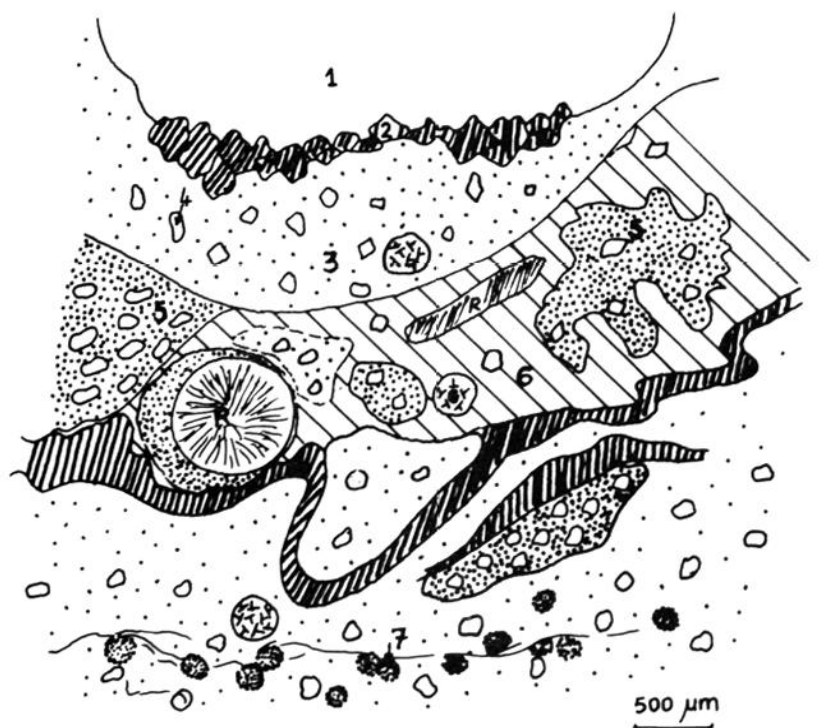

Fig. 5 : COUPE DE L'ENCROUTEMENT CALCAIRE (OL 7) 1 : galet - 2 : sparite palissadique - 3 : micrite claire grise 4: quartz - 5: micrite brune sombre - 6 : microsparite 7 : micronodules - $\mathbf{R}$ : reliques - $\mathrm{G}:$ glauconie.
$-40 \mathrm{~cm}$ de croûte calcaire (OL 8) saumon $(7,5$ YR 6/6) débitée en miches épaisses de 2 à $3 \mathrm{~cm}$, contigües ou séparées, en 3 à 6 niveaux superposés, séparés par $3 \mathrm{~cm}$ de limons. Le squelette est essentiellement formé de grains de quartz non craquelés et de taille variable $(50 \mu \mathrm{m}$ de diamètre moyen) pouvant atteindre $60 \%$ de la roche totale. Les feldspaths sont toujours présents mais rares et la glauconie également, avec 2 à $4 \%$ de l'ensemble du squelette. Ici aussi, les grains de quartz sont bien corrodés et remplacés par la microsparite, les bordures intactes gardent toujours des reliques d'argiles et/ou d'oxydes de fer. On retrouve les reliques des roches anciennes ainsi que les grains de sparite dejà signalés dans les niveaux limoneux. Le plasma est une micrite brune plus ou moins argileuse, le plus souvent orientée ou structurée en nodules ou micronodules. Dans certains cas, l'orientation du plasma provoque des concentrations inégalement réparties des grains du squelette. En effet, ceux-ci peuvent dessiner de petits chenaux ou former des microlits suivant la sédimentation micritique (fig. 6). 


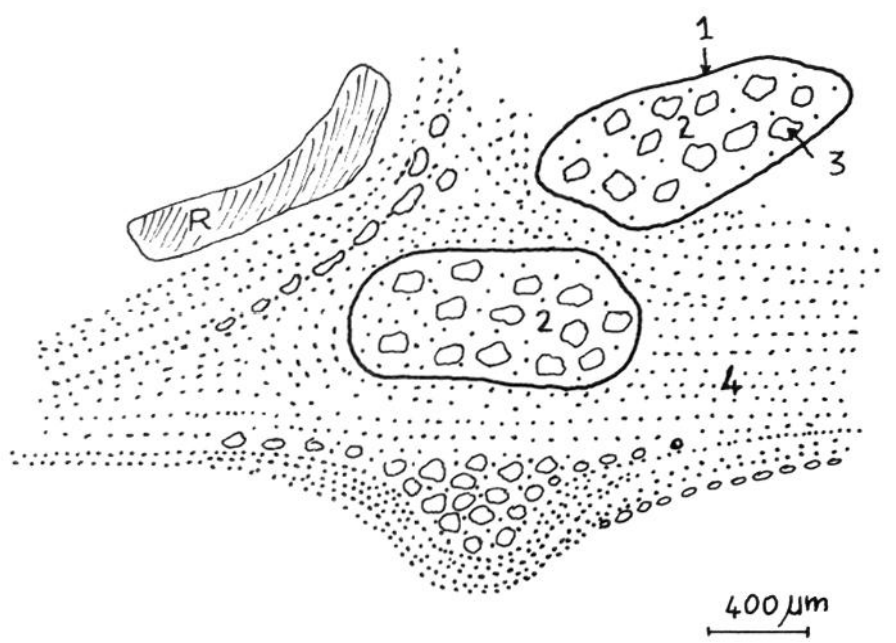

Fig. 6 : COUPE DE LA CROUTE CALCAIRE INFERIEURE (OL 8) $1:$ nodules $-2:$ micrite grise $-3:$ quartz $-4:$ micrite gris-jaunâtre orientée - $\mathbf{R}$ : reliques. (aspect nodulaire, dispersées dans une "pâte» gypseuse. Ce gypse, qui constitue plus de $80 \%$ de la roche totale, englobe également des grains de quartz ou de glauconie lâchement dispersés.

La quatrième séquence comprend :

$-30 \mathrm{~cm}$ de sable limoneux (OL 13) blanc (10YR 8/4), à structure feuilletée, riche en $\mathrm{CaO}$ et surtout en $\mathrm{Al}_{2} \mathrm{O}_{3}$, peu sulfaté et peu siliceux (fig. 4).

-20 à $30 \mathrm{~cm}$ de croûte calcaire beige saumon (OL 14) (5YR 8/3), en miches ou en gros feuillets séparés par des nodules issus de la fragmentation de la croûte, de composition géochimique très comparable à celle de la croûte inférieure (fig. 4). On y observe deux catégories de microfaciès :

-un plasma non orienté : il correspond à une micrite gris clair, plus ou moins homogène, dans laquelle baignent des grains de squelette (quartz, feldspaths, glauconie). Ici également, les grains de quartz sont corrodés et remplacés par la microsparite ; dans les golfes de corrosion subsistent parfois les reliques d'argiles et/ou de fer ;

-un plasma orienté : il est constitué par une micrite gris foncé orientée qui renferme quelques grains de quartz de petite taille. Cette micrite peut alterner avec des lits également orientés de grains de squelette. Cette zone correspond au durcissement maximum de la croûte. Au fort grossissement (200 fois), ce plasma orienté forme plutôt un tapis dessinant des figures fibroradiées en oursins, en choux-fleurs ou en feuillets rappelant bien l'aragonite. Il s'agit vraisemblablement de tapis algaires.

-10 à $20 \mathrm{~cm}$ d'un deuxième faciès de croûte calcaire, discontinu, beige (5YR 8/4), plus ou moins lité, avec des fragments de croûte en feuillets plats.

- reg dans un limon sableux.

\section{B. Les variations latérales}

Dans l'axe des talwegs, vers l'amont, la coupe tend à se simplifier. En effet, si le conglomérat basal reste très constant, les deux autres peuvent disparaître. Par contre, la concentration en gypse correspondant à OL 12 et la croûte calcaire sommitale (OL 14) ont aussi une valeur régionale. Latéralement, les versants sont couverts de cette croûte calcaire sommitale sous laquelle subsiste la croûte gypseuse qui vient affleurer parfois dans le tiers supérieur (fig. 3), surtout à orientation ouest, ou elle peut venir reposer directement sur le calcaire campanien.

\section{Les évolutions à l'échelle du Pléistocène moyen}

\section{1) L'évolution minéralogique}

L'analyse minéralogique totale révèle la présence du quartz, de la calcite, du gypse et du feldspath (fig. 7), confirmant l'analyse microscopique. Le quartz et la calcite sont omniprésents, alors que le gypse disparaît à la base et au sommet et que le feldspath est limité à la partie moyenne. D'une manière générale, l'augmentation de la proportion de gypse se fait au détriment de celle de la calcite et indépendamment de celle du quartz. 


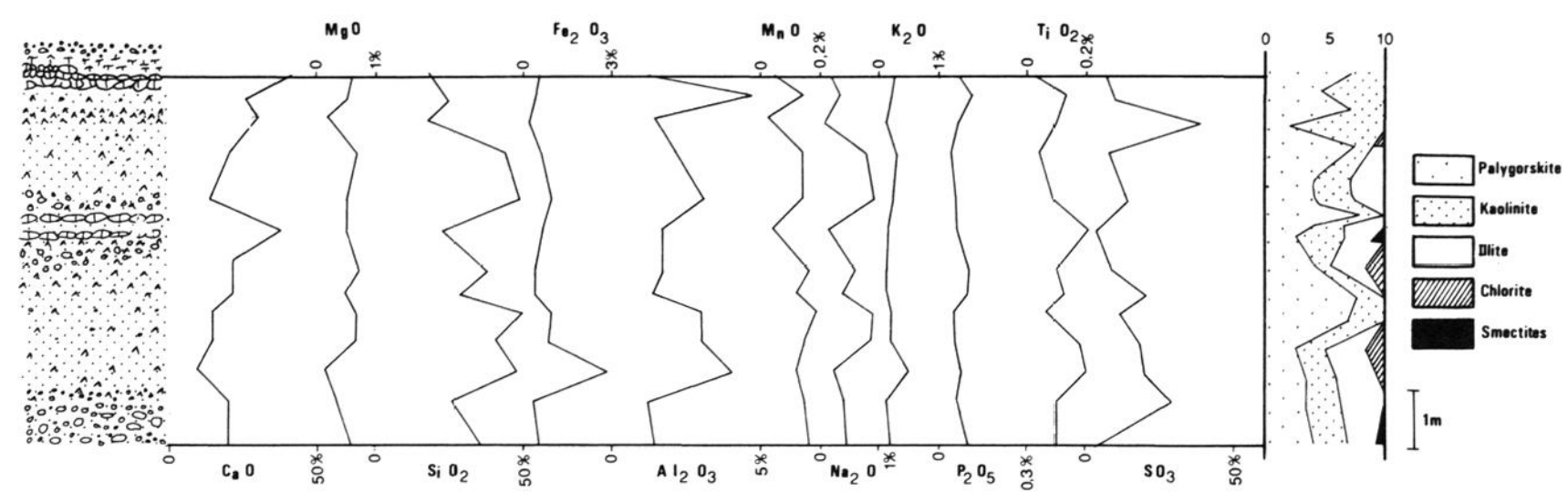

Laboratorre de cartographie de la faculte des Sciences Humaines et Sociales de Tunis

Fig. 7 : COMPOSITION MINERALOGIQUE DES DEPOTS DE LA HAUTE TERRASSE

La subdivision de la coupe en quatre parties, mise en évidence par le découpage en séquences, est confirmée par l'étude des minéraux argileux. En effet, les argiles montrent une association variable de paly. gorskite, kaolinite, illite, chlorite et smectites (fig. 4), cortège pratiquement semblable à celui des limons de Matmata (REGAYA, 1983). Les smectites et les chlorites sont faiblement représentées aux côtés du cortège palygorskite-kaolinite-illite. Les chlorites $(0,5 / 10$ à $1 / 10)$ se trouvent à trois niveaux distincts, dans les limons juste au-dessus du conglomérat de la première séquence, dans la matrice du conglomérat de la deuxième séquence et enfin juste à la base de la croûte gypseuse de la troisième séquence. Les smectites $(0,5 / 10$ à $1 / 10)$ sont présentes à la base de la première séquence et dans la croûte calcaire de la deuxième séquence. La kaolinite est omniprésente le long du profil, sa teneur a une valeur moyenne comprise entre $2 / 10$ et $3 / 10$, exceptée la matrice du conglomérat de la deuxième séquence $(1,5 / 10)$ et la croûte gypseuse de la troisième séquence. En effet, juste au-dessus des conglomérats de chacune de ces séquences, elle a des teneurs comprises entre 2,5/10 et $3,5 / 10$, et plus haut et juste en dessous de la croûte (calcaire ou gypseuse) sa valeur est maximale $(7 / 10$ à $7,5 / 10$ ) ; enfin, au sommet, au niveau même de la croûte, cette valeur fléchit à $4,5 / 10$ ou même à $2 / 10$. L'illite a des valeurs comprises entre $3 / 10$ et $5 / 10$; elle disparait complètement quand les valeurs de la palygorskite sont maximales.

L'inventaire minéralogique de la fraction argileuse de cette terrasse est franchement hétérogène et s'oppose à la nette dominance des smectites des séries du Crétacé supérieur du Dahar. La présence d'un mélange de cinq types d'argiles reflète une allochtonie probablement lointaine d'une partie de ces argiles: la kaolinite, la chlorite, l'illite et, à un moindre degré, la palygorskite. Par contre, pour les smectites, une reprise à partir du calcaire campanien peut s'envisager. La variation des teneurs en palygorskite et en illite paraît significative et évolutive. En effet, si l'on prend la première séquence comme exemple, on constate qu'au-dessus du conglomérat les limons contiennent des teneurs en palygorskite et en illite presque égales $(2,5 / 10$ à $3 / 10$ pour la première et $3,5 / 10$ pour la seconde); dans la partie médiane juste au-dessous de la croûte, le taux de palygorskite croît sensiblement à 7,5/10, en revanche, l'illite disparait complètement ; plus haut, c'est-à-dire dans la croûte même, la palygorskite fléchit à $6 / 10$ alors que l'illite réapparaît à $1 / 10$. Cette configuration semble se répéter pour les autres séquences. Ceci nous incite à croire qu'une partie au moins de la palygorskite pourrait être néoformée par agradation au dépens de l'illite (TRAUTH N., 1977) (et des smectites) mais cela est fonction du degré de confinement, lui-même tributaire des conditions climatiques environnantes. Ceci n'exclut pas qu'une fraction de la palygorskite soit détritique (COUDE-GAUSSEN et al., 1984).

\section{2) Les corrélations géochimiques}

Dans cette coupe, $\mathrm{SiO}_{2}$ et $\mathrm{SO}_{3}$, d'une part, $\mathrm{SiO}_{2}$ et $\mathrm{CaO}$, d'autre part, sont corrélés négativement, alors que $\mathrm{Al}_{2} \mathrm{O}_{3}$ et $\mathrm{SiO}_{2}$ sont corrélés positivement.

Pour $\mathrm{SiO}_{2}$ et $\mathrm{SO}_{3}$, le coefficient de corrélation de BRAVAIS-PEARSON est $\mathrm{r}=-0,701$, significatif à $98 \%$, le pourcentage de variance expliqué est d'environ $49 \%$. Pour $\mathrm{SiO}_{2}$ et $\mathrm{CaO}$, le même coefficient de corrélation est $\mathrm{r}=-0,827$, significatif à $98 \%$ et le pourcentage de variance expliqué est d'environ $68 \%$. Enfin, pour $\mathrm{Al}_{2} \mathrm{O}_{3}$ et $\mathrm{SiO}_{2}$, le coefficient est $\mathrm{r}=0,694$, toujours significatif à $98 \%$. Le pourcentage de la variance expliqué est d'environ $48 \%$. 


\section{III - DISCUSSION ET INTERPRETATION PALEOCLIMATIQUE}

\section{A. L'origine du matériel}

\section{1) Le quartz}

La petite taille des grains de quartz ( $80 \mu \mathrm{m}$ de diamètre maximum) et leur absence des séries crétacées sous-jacentes sont deux critères suffisants pour une origine lointaine et éolienne de ce minéral. Toutefois, les preuves de l'épigénie des grains de quartz par la calcite, d'une part, et la présence des enduits argileux et/ou d'oxydes de fer des golfes de corrosion montrent que ce sédiment a connu au moins une phase de pédogenèse avant son dépôt dáns la terrasse. Ceci implique qu'il appartient à des formations loessiques accumulées à l'amont du bassin-versant de l'oued Ahmadi, dans les Monts de Matmata.

\section{2) Le gypse}

Compte tenu du fait que le bassin-versant de l'oued Ahmadi ne contient pas de sulfate, la corrélation inverse entre $\mathrm{SiO}_{2}$ et $\mathrm{SO}_{3}$ confirme les observations microscopiques montrant que le quartz et le gypse (c'est-à-dire l'essentiel des sulfates) ne se déposent pas au même moment, encore qu'une arrivée simultanée soit parfois possible puisque la corrélation n'explique qu'environ $49 \%$ de la variance. Une autre interprétation serait de considérer que $\mathrm{SiO}_{2}$ et $\mathrm{SO}_{3}$ se concentrent alternativement. On peut la rejeter pour l'essentiel car, si des phénomènes d'épigénisation du quartz par le gypse ont été signalés ailleurs (STRAKHOV, 1957), il n'en a pas été observés dans la terrasse de l'oued Ahmadi. Si le quartz provient des limons de Matmata remaniés par le ruissellement, par contre, le gypse arrive sous forme de poussières qui peuvent être reprises et redistribuées secondairement dans les pores.

\section{3) La calcite}

Dans la plupart des cas, la calcite, surtout micritique, constitue le plasma qui sert de liant des grains du squelette. Cette calcite semble provenir de l'environnement carbonaté puis, véhiculée sous forme de bicarbonate de calcium par les cours d'eau et les nappes phréatiques, elle parvient au niveau des limons où elle précipite et pourrait former, aux niveaux les plus bas, des dalles de croûte calcaire. $\mathrm{La}$ corrélation négative entre $\mathrm{SiO}_{2}$ et $\mathrm{CaO}$ et l'absence de corrélation entre $\mathrm{SO}_{3}$ et $\mathrm{CaO}$ confirment que la silice et le calcium arrivent (ou se concentrent) à des moments différents et ne s'opposent pas à une origine différente de ces deux éléments. La part éventuelle de poussières calcaires est donc négligeable en regard des apports de l'oued et des nappes.

Au total, se regroupent donc, dans la terrasse, trois types de matériel différents :

- du matériel calcaire d'origine fluviatile directe,

- du matériel gypseux d'origine éolienne directe,

- du matériel siliceux d'origine éolienne primaire, remanié par le ruissellement.

\section{B. Régime des vents et paléoclimatologie}

La source la plus vraisemblable du gypse est constituée par le chott el Jerid, origine, avec le Fedjaj et le Gharsa, de plusieurs générations de croûtes reconnues par R. COQUE (1962). On sait maintenant (FONTES et al., 1987a, 1987b) que des lacs se sont succédés dans le bassin des Grands Chotts au cours du Pléistocène moyen, séparés par des périodes d'assèchement favorables à la déflation éolienne. On peut donc envisager, à titre d'hypothèse en attendant des datations précises, que les fluctuations du taux de sulfates dans la coupe de l'oued Ahmadi sont en relation avec celles de l'hydrologie du bassin des Grands Chotts. Ceci suppose un apport de poussières gypseuses par vents de secteur Nord-Ouest. Actuellement si, dans l'ensemble du Sud tunisien, la connaissance du régime des vents a progressé récemment (KHAZNI, 1986), il est encore très mal connu au sud-est du chott el Jerid. Cependant, en hiver, les vents d'Ouest à Sud-Ouest dominent dans l'ensemble, en particulier à Gabès et Médenine, les deux stations les plus proches, dont $1 / 3$ à $1 / 2$ à vitesse comprise entre 5 et $9 \mathrm{~m} / \mathrm{s}$. En été, dominent les vents de secteur Nord-Est à Est avec une forte proportion de vents à vitesse inférieure à $5 \mathrm{~m} / \mathrm{s}$. Les saisons intermédiaires connaissent des situations plus complexes sauf à Medenine et Remada où persistent les vents d'Ouest (KHAZNI, 1986), ainsi qu'à Matmata (MTIMET, 1983).

Le régime des vents qu'on peut reconstituer pour la partie du Pléistocène moyen représentée par la terrasse ne semble pas radicalement différent de l'actuel. Tout juste faut-il envisager, pour le chott el Jerid, une composante plus méridienne que dans les vents actuels de Tozeur dominés par le courant zonal d'Ouest ou d'Est (KHAZNI, 1986).

Le matériel quartzeux, bien que d'origine éolienne, peut difficilement renseigner sur la direction des paléovents puisque remaniés des cuvettes des Monts de Matmata drainées vers l'Ouest, après avoir subi une 
pédogenèse. En effet, on ne peut estimer le temps nécessaire au dépôt, puis à la pédogenèse et enfin au remaniement fluviatile de ces limons quartzeux. Toutefois, deux provenances possibles sont à évoquer, soit les Grands Chotts, soit le Grand Erg oriental (COUDE-GAUSSEN et al., 1983 ; BALLAIS, BALLAND, 1983 ; YAALON, 1986).

Plus généralement, par comparaison avec le climat aride inférieur actuel (GIESSNER, 1979) et la dynamique actuelle (rares crues de l'oued Ahmadi dans un lit majeur très large à gros galets et quelques nebkas à Raetama retam), l'apport de conglomérats indique des conditions peu différentes. A l'opposé, le transport et le dépôt de limons prélevés dans la partie amont du bassin suppose des écoulements à trajets plus longs et à compétence plus faible qu'actuellement, soit un climat à humidité plus importante et mieux répartie dans l'année. Les croûtes calcaires supposent une humidité suffisante pour la mobilisation des carbonates, mais il faut distinguer ici selon le type croûte. La croûte inférieure présente une base à genèse plutôt phréatique et un sommet plutôt sédimentaire, nécessitant une humidité locale plus forte qu'actuellement. La croûte supérieure, d'origine biologique, ne suppose qu'une humidité locale réduite. Enfin, la croûte gypseuse suppose des conditions très proches de l'Actuel avec une déflation sur les Grands Chotts et le piégeage des poussières par une steppe qui serait bien plus dense si elle n'était soumise au surpâturage. De telles concentrations gypseuses actuelles sont connues dans des conditions comparables, par exemple dans les Ziban (BALLAIS et al., 1987).

L'organisation des formations en quatre séquences sédimentaires pourrait traduire l'existence de quatre oscillations climatiques. En effet, dans un climat qui reste, dans l'ensemble, aride, des pulsations légèrement plus humides se sont produites comme l'attestent l'accumulation des limons et la genèse des croûtes calcaires. Le Pléistocène moyen apparaît donc comme une période plus complexe qu'on ne l'envisageait jusqu'à maintenant puisqu'on peut y déceler au moins quatre fluctuations climatiques. En outre, la genèse d'une croûte calcaire postérieurement à celle d'une croûte gypseuse indique un renversement épisodique de la tendance généralisée à l'aridification du climat pendant le Quaternaire.

\section{IV - CONCLUSION}

Ainsi, la haute terrasse de l'oued Ahmadi résulte de l'accumulation, au Pléistocène moyen, d'apports fluviatiles de conglomérats calcaires et de limons quartzeux repris de dépôt loessiques, d'apports de carbonates en solution et d'apports de poussières gypseuses venus d'aval, par voie aérienne.

La coupe de cette terrasse montre :

- l'âge minimum pléistocène moyen de la mise en place d'une grande partie des limons de Matamata,

- la provenance depuis le bassin des Grands Chotts des poussières gypseuses,

- la complexité du Pléistocène moyen. Cette complexité, confirmée par l'identification récente de plusieurs phases lacustres, est accrue par la mise en évidence de fluctuations climatiques.

\section{REFERENCES BIBLIOGRAPHIQUES}

BALLAIS J.-L. (1984), - Recherches géomorphologiques dans les Aurès (Algérie), A.N.R.T. Lille III, 2 vol., VII, $626 \mathrm{p}$.

BALLAIS J.-L. et BALLAND V. (1983). - Discussion sur l'origine du «loess» pléistocène de Téchine (Sud tunisien), Bull. Soc. Géol. Fr., (7), t. XXV, n 4, pp. 635-636.

BALLAIS J.-L., DUMONT J.-L., LE COUSTUMER M.-N. et LEVANT M. (1987). - Sédimentation éolienne, pédogenèse et ruissellement au Pléistocène supérieur-Holocène dans les Ziban (Algérie), sous presse.

BEN OUEZDOU H. (1986). - Essai de corrélation des formations quaternaires continentales et marines dans les alentours du golfe de Gabès. Géodynamique 1 (2), pp. 81-95.

BEN OUEZDOU H. (1987). - Etude morphologique et stratigraphique des formations quaternaires des alentours du golfe de Gabès, Revue des Sciences de la Terre, Mémoire, vol. 5, Tunis, $165 \mathrm{p}$.

COQUE R. (1962). - La Tunisie présaharienne, étude géomorphologique, A. Colin, Paris, 476 p.

COUDE-GAUSSEN G., MOSSER C., ROGNON P. et TOURENQ J. (1982). - Une accumulation de loess du Pléistocène supérieur dans le Sud tunisien : la coupe de Téchine, Bull. Soc. Géol. Fr., t. XXIV, n², pp. 283-292.

COUDE-GAUSSEN G., LE COUSTUMER M.-N. et ROGNON P. (1984). - Paléosols d'âge pléistocène supérieur dans les loess de Matmata (Sud tunisien), Sciences géol., Strasbourg, 37, 4, pp. 359-386. 
FONTES J.-C., GASSE F. et al. (1987a), - Programme Palhydaf. Etat d'avancement, janvier 1987. Premier méridien : Sud Tunisie-Sud Niger, Séminaire Paléolacs Paléoclimats, Orstom, $4 \mathrm{p}$.

FONTES J.-C., GASSE F. et ZOUARI K. (1987b). - Lacustrine sediments from Wadi el Akarit and chott Fedjej (South Tunisia) since 140000 B.P. (Palhydaf Project site 1), Symposium on Paleoclimatic and environmental reconstructions from lacustrine basins, Strasbourg, 2 p.

GHANMI M. (1986). - Carte géologique de Tamezret au 1/100 000, sous presse.

GIESSNER K. (1979). - Die Klima - und Phytoökologische Gliederung Tunesiens, Würzburger Geographische Arbeiten, Heft 49, pp. 199-235.

KHAZNI M. (1986). - Statistique sur le vent dans le Sud tunisien, Séminaire international sur la lutte contre la désertification, Jerba, $9 \mathrm{p}$.

MTIMET A. (1983). - Contribution à l'étude pédologique des limons de Matmata (Sud tunisien), Thèse 3ème cycle, Paris VI, 2 rol., 183 p.

REGAYA K. (1983). - Etude géologique de la formation des limons de Matmata (Sud tunisien), Thèse 3ème cycle, Aix-Marseille, $121 \mathrm{p}$.

STRAKHOV M. (1957). - Méthode d'étude des roches sédimentaires, Serv. Inf. Géol., Annales vol. $1, \mathrm{n}^{\circ} 35$.

TRAUTH N. (1977). - Argiles évaporitiques dans la sédimentation carbonatée continentale et épicontinentale tertiaire. Bassins de Paris, de Mormoiron et de Salinelles (France), Jbel Ghassoul (Maroc), Mémoires Sciences géol., Strasbourg, $\mathrm{n}^{\circ} 49,195 \mathrm{p}$.

YAALON D. (1986). - Sahara dust and desert loess : effect on surrounding soils, Bull. liaison ASEQUA, Dakar, $\mathrm{n}^{\circ} 76, \mathrm{pp} .115-117$.

ZOUARI H., KAMMOUN Y. et REGAYA K. (1986). - Carte géologique de Matmata au 1/100 000, O.N.M. et I.N.R.S.T., Tunis. 